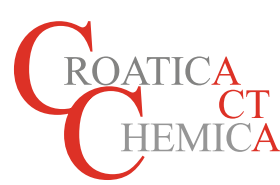

\title{
Crystal Growth Mechanism of Vaterite in the Systems Containing Charged Synthetic Poly(Amino Acids)
}

\author{
Branka Njegić Džakula, ${ }^{1, *}$ Giuseppe Falini, ${ }^{2}$ Damir Kralj ${ }^{1, \#}$
}

\author{
${ }^{1}$ Laboratory for Precipitation Processes, Division of Materials Chemistry, Ruđer Bošković Institute, Bijenička cesta 54, HR-10000 Zagreb, Croatia \\ 2 Dipartimento di Chimica "Giacomo Ciamician", Universitá di Bologna, Via Selmi 2, 40126 Bologna, Italy \\ * Corresponding author's e-mail address: bnjeg@irb.hr \\ \# Corresponding author's e-mail address: kralj@irb.hr
}

RECEIVED: January 18, 2018 * REVISED: April 17, 2018 * ACCEPTED: April 17, 2018

THIS PAPER IS DEDICATED TO PROF. MLADEN ŽINIĆ ON THE OCCASION OF HIS 70TH BIRTHDAY

\begin{abstract}
Negatively ionisable poly-L-glutamic acid (pGlu) and poly-L-aspartic acid (pAsp), considered as analogues of the naturally occurring acidic macromolecules involved in biomineralization processes, were used as additives in the calcium carbonate precipitation systems in order to investigate their interactions with the vaterite crystallites. Poly-L-lysine (pLys), a positively ionisable poly(amino acid), was also used in order to elucidate the impact of the side chain charge. The growth kinetics of vaterite was found parabolic, indicating that the integration of growth units into the spiral step at the vaterite crystal surfaces is the rate-determining mechanism. The presence of small amounts of pGlu and pAsp inhibited the crystal growth. At the highest concentrations of both acidic macromolecules the exponential rate law was observed, which indicates the surface nucleation as the rate controlling mechanism. The addition of pLys in the range of applied concentrations did not significantly influence the crystal growth of the vaterite. Thus, the kinetic results, corroborated by morphological observations, pointed out to the significance of the negative charge of the side chains of selected polypeptides in the interfacial interactions with mineral planes.
\end{abstract}

Keywords: crystal growth of vaterite, biomineralization, calcium carbonate, poly-L-glutamic acid, poly-L-aspartic acid.

\section{INTRODUCTION}

C ALCITE and aragonite are the thermodynamically most stable calcium carbonate polymorphs, which can be frequently found in biomineralized tissues of many invertebrate organisms. Calcium carbonate also forms vaterite, the least stable polymorph, and three hydrated modifications: calcium carbonate monohydrate (CCM), calcium carbonate hexahydrate $(\mathrm{CCH})$ and amorphous calcium carbonate (ACC). All modifications can be found in nature, but due to its instability, vaterite is not wide-spread: it can be found in sediments ${ }^{[1]}$ and metamorphic rocks ${ }^{[2]}$ but also in some biological systems, like in the hard tissues of fish otoliths, ${ }^{[3]}$ crustacean statoliths, ${ }^{[4]}$ ascidian skeleton ${ }^{[5]}$ or in the fresh water pearls. ${ }^{[6]}$ Vaterite is found to be an important precursor in several calcium carbonate forming processes. However, the crystallization pathway of vaterite is unclear and it is very difficult to obtain large and pure single-crystals of vaterite. For this reason, the exact crystal structure of vaterite is also not solved yet, although some recent studies proved that it is composed of two different structural units. ${ }^{[7]}$ The investigations of vaterite nucleation, growth and transformation can contribute to the understanding of the specific mechanisms of biomineralization, but also to the understanding of the processes of crystal nucleation and growth in general. ${ }^{[8]}$

A considerable amount of research on the processes occurring at the organic-inorganic interfaces and of calcium carbonates, related to the biomineralization or bioinorganic chemistry, has already been performed. ${ }^{[9-22]}$ Thus, the model studies on crystallization at the organic-inorganic interfaces, carried out in vitro, typically use the macromolecules extracted from biogenic crystals ${ }^{[15,17-19,21-30]}$ or synthetic macromolecules, ${ }^{[9,14,15,31-36]}$ which are considered 
to be their analogues. In addition, the experimental data indicate that the metastable modifications like ACC, are the first mineral phases that appear during the formation of shells, thus acting as a reservoir of constituting ions for subsequent formation of stable modifications. On the other hand, in vitro experiments of spontaneous ACC precipitation indicate that its transformation into stable polymorph(s), calcite and aragonite, practically always proceeds through the formation of vaterite as an intermediate modification. ${ }^{[37-40]}$ During the final period of such precipitation which started at high initial supersaturations, the vaterite transforms into calcite by a solution-mediated process. ${ }^{\left[{ }^{14,42]}\right.}$ The vaterite transformation has been comprehensively studied previously[41] in the systems in which it spontaneously forms in the moderately supersaturated calcium carbonate solutions. However, if vaterite is removed from the solution and dried, it remains stable even at the temperatures up to about $456^{\circ} \mathrm{C}$. ${ }^{[43]}$

This work complements previous investigations on the interactions between the crystal surfaces of calcium carbonate polymorphs and the charged synthetic poly(amino acids). ${ }^{[34,35]}$ Previously, we investigated in details the nature and the extent of interactions between the well-defined calcite crystallites and the synthetic poly(amino acids), poly-L-glutamic (pGlu) and poly-L-aspartic (pAsp) acids, considered as analogues of the naturally occurring acidic macromolecules responsible for biomineralization process in mollusc shells. In addition, spontaneous precipitation of $\mathrm{CaCO}_{3}$ in the systems in which several solid phases can nucleate was investigated in the presence of the selected macromolecules. The positively ionisable poly-Llysine (pLys) was also used in those investigations, in order to elucidate the role of the side chain charge in the interaction with a mineral substrate.

In this work, structurally and morphologically welldefined vaterite seed crystals have been used for inoculation of moderately supersaturated calcium carbonate solutions, which contained the selected poly(amino acids), in order to analyse kinetics of the crystal growth process. ${ }^{[44,45]}$ By using the kinetic data and morphological analyses, the vaterite crystal growth rate mechanisms have been revealed, as well as the mode and extent of vaterite / poly(amino acids) interactions. We are confident that these results will contribute to a deeper understanding of the basic principles of mineral phase/organic macromolecule interactions, which are involved in biomineralization, particularly in the early stages of the process.

\section{EXPERIMENTAL}

Reactant solutions, $\mathrm{CaCl}_{2}$ and $\mathrm{Na}_{2} \mathrm{CO}_{3}$, were prepared from analytical grade chemicals (both Merck) and deionized water of high quality (conductivity $<0.055 \mu \mathrm{S} \mathrm{cm}^{-1}$ ). Stock solutions of poly-L-glutamic acid (Miles-Yeda Ltd.), poly-Laspartic acid (Sigma) and poly-L-lysine (Sigma), all $c=$ $100 \mathrm{ppm}$, were prepared by using the chemicals of average molecular masses, $M_{\mathrm{r}}=12300 \mathrm{~g} \mathrm{~mol}^{-1}, M_{\mathrm{r}}=11100 \mathrm{~g} \mathrm{~mol}^{-1}$, and $M_{\mathrm{r}}=8800 \mathrm{~g} \mathrm{~mol}^{-1}$, the corresponding numbers of their monomer units being 95, 96 and 69, respectively. The experiments were performed in a $400 \mathrm{~cm}^{3}$ thermostated double-walled glass vessel tightly closed by a Teflon cover, in order to minimize contamination with carbon dioxide from the air. The vaterite seed crystals and the appropriate concentration of the poly(amino acids) (pAsp, pGlu or pLys) were suspended into the $200 \mathrm{~cm}^{3}$ of $\mathrm{Na}_{2} \mathrm{CO}_{3}$ solution. The so prepared carbonate suspension was homogenized by ultrasonication for $40 \mathrm{~s}$, before identical volume of $\mathrm{CaCl}_{2}$ solution was poured into the system. The initial reactant concentrations in the system were equimolar and were $c_{\mathrm{i}}\left(\mathrm{CaCl}_{2}\right)=c_{\mathrm{i}}\left(\mathrm{Na}_{2} \mathrm{CO}_{3}\right)=1.0 \times 10^{-3} \mathrm{~mol} \mathrm{dm}^{-3}$. The vaterite seed used for the crystal growth experiments, were prepared according to the procedure previously described. ${ }^{[35]}$ The initial mass concentrations of the vaterite seed crystals was $\rho_{\text {vaterite }}=250 \mathrm{mg} \mathrm{dm}{ }^{-3}$. The range of poly(amino acid) concentrations was: $0.3 \mathrm{ppm}<c_{\mathrm{i}}$ (pAsp, pGlu) $<1.0 \mathrm{ppm}$ and $0.2 \mathrm{ppm}<c_{\mathrm{i}}$ (pLys) $<4.0 \mathrm{ppm}$ (or in $\mu \mathrm{mol} \mathrm{dm}{ }^{-3}, 3 \mu \mathrm{mol} \mathrm{dm}{ }^{-3}<c_{\mathrm{i}}$ (pAsp, pGlu) $<9 \mu \mathrm{mol} \mathrm{dm}^{-3}$ and $2 \mu \mathrm{mol} \mathrm{dm}{ }^{-3}<c_{\mathrm{i}}$ (pLys) $<31 \mu \mathrm{mol} \mathrm{dm}^{-3}$ ). During the experiments, the system was continuously stirred at constant rate by means of a Teflon-coated magnetic stirring bar and the temperature was maintained at $25{ }^{\circ} \mathrm{C}$. The systems containing identical initial reactant concentrations and volume, as well as identical mass concentration of vaterite seed crystals, but without poly(amino acids) addition, were used as control.

The propagation of the process was continuously monitored by measuring $\mathrm{pH}$ of the solution as a function of time by means of a combined glass-calomel electrode (GK 2401 C) connected to a digital pH meter (PHM 290, Radiometer). During each run aliquots of suspension were periodically taken from the system and filtered through $0.22 \mu \mathrm{m}$ membrane filters. At the end of each experiment, the remaining volume of suspension was filtered through a $0.22 \mu \mathrm{m}$ membrane filter, washed with small volumes of water, and dried at $105^{\circ} \mathrm{C}$.

The mineralogical composition of the dried crystals was determined by using the X-ray diffraction of powdered samples (PANanalytical X' PertPRO, powder diffractometer equipped with a monochromator on the diffracted beam, using $\mathrm{Cu} \mathrm{K \alpha}$ radiation $(40 \mathrm{~mA}, 40 \mathrm{kV}))$ and by FT-IR spectroscopy (FT-IR Mattson spectrometer, Genesis Series). The specific surface area, SSA, of crystals was determined by the multiple BET method (Micromeritics, Gemini), using nitrogen gas as adsorbate, and the morphology was observed by using scanning electron microscopy, SEM (XL20, Philips). 


\section{Treatment of Data}

The recorded $\mathrm{pH}$ data were used for calculations of the initial solution composition and the solution composition at any time of the vaterite crystal growth process. In addition to $\mathrm{pH}$, the known total calcium chloride and sodium carbonate concentrations, added initially to the system, enabled the calculations of molar concentrations and activities of the ionic species assumed to be present in solution in relevant amounts: $\mathrm{H}^{+}, \mathrm{OH}^{-}, \mathrm{CO}_{3}{ }^{2-}, \mathrm{HCO}_{3}{ }^{-}, \mathrm{NaCO}_{3}{ }^{-}, \mathrm{CaCO}_{3}{ }^{0}$, $\mathrm{CaHCO}_{3}{ }^{+}, \mathrm{CaOH}^{+}, \mathrm{Ca}^{2+}, \mathrm{Na}^{+}$, and $\mathrm{Cl}^{-}$. The detailed calculation procedure, which takes into account the respective protoIytic equilibria and equilibrium constants as well as mass and charge balance equations, is shown previously. ${ }^{[34,40,41,46-48]}$ The impact of the poly(amino acid) addition on the solution composition was estimated considering the ionic species and ionic equilibria associated to this case. ${ }^{[49]}$ However, that poly(amino acids) were added into the system at very low concentrations so their impact on solution composition was neglected. When the highest concentration of pGlu ( $c=1 \mathrm{ppm}), \operatorname{pAsp}(c=1 \mathrm{ppm})$ or plys ( $c=4 \mathrm{ppm}$ ) was applied, the initial supersaturation changed less than $1 \%$. At that, the poly(amino acids) were assumed to behave as the respective monomer units. Supersaturation was expressed as a relative supersaturation, $S-1$, and the saturation ratio as, $S=\left(\Pi / K_{\mathrm{sp}}^{0}\right)^{1 / 2}$. In the above expressions, $\Pi=a\left(\mathrm{Ca}^{2+}\right) a\left(\mathrm{CO}_{3}{ }^{2-}\right)$, is the ion activity product, while $K_{\mathrm{sp}}{ }^{0}=1.221 \times 10^{-8}$, is the thermodynamic equilibrium constant of dissolution of vaterite at $25^{\circ} \mathrm{C}$.

The crystal growth rate of vaterite, $-\mathrm{dCa} a_{\text {tot }} / \mathrm{d} t$, was determined by numerical differentiation of the total concentration of dissolved calcium species, $\mathrm{Ca}_{\text {tot }}$, and it was expressed as a function of supersaturation at a given moment:

$$
v_{\mathrm{g}}=-\mathrm{d} C a_{\mathrm{tot}} / \mathrm{d} t=K A(S-1)^{n}
$$

where $K$ is a crystal growth rate constant, $A$ is a surface area of precipitate, $A=f V^{2 / 3}$; $f$ is a shape factor, and $n$ is the apparent order of the reaction.

The precipitate volume $(\mathrm{V})$ was given by

$$
V=c_{p p t} V_{m}
$$

where $c_{\mathrm{ppt}}$ is the concentration of precipitated calcium carbonate and $V_{\mathrm{m}}$ is the specific molar volume of vaterite. The concentration, $c_{\mathrm{ppt}}$, was determined by subtracting the calculated total concentration, $c_{\text {tot }}$, of calcium or carbonate species in the closed system from the known initial concentration of calcium chloride or sodium carbonate.

From the above equations, the following equation for the linear growth rate of vaterite was derived:

$$
v_{\mathrm{g}}=k_{\mathrm{n}} \mathrm{c}_{\mathrm{ppt}}{ }^{2 / 3}(S-1)^{n}
$$

where the crystal growth rate constant is expressed by $k_{\mathrm{n}}$. In the calculations the number and shape of the vaterite crystals were assumed to be constant during the experiment.

\section{RESULTS AND DISCUSSION}

In order to ensure reproducibility of kinetic measurements and to avoid errors caused by a possible minor difference in physical-chemical properties of vaterite seeds used for inoculation, the same batch of crystals was used throughout all the experiments. Figure 1 . shows the scanning electron micrograph of the used spherical vaterite particles. The FT-IR spectroscopy and X-ray diffraction analyses of dry samples confirmed that vaterite was the unique solid phase prepared and used in the experiments. The specific surface area of the so-prepared vaterite crystals was $4.7 \mathrm{~m}^{2} \mathrm{~g}^{-1}$.

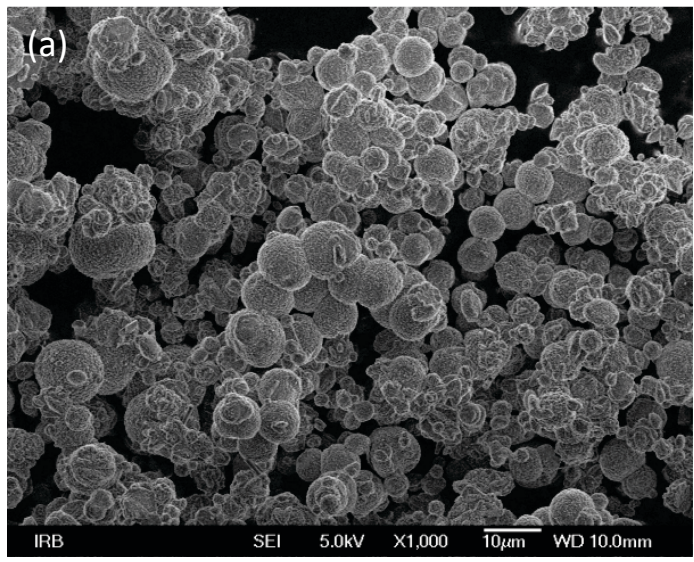

(b)

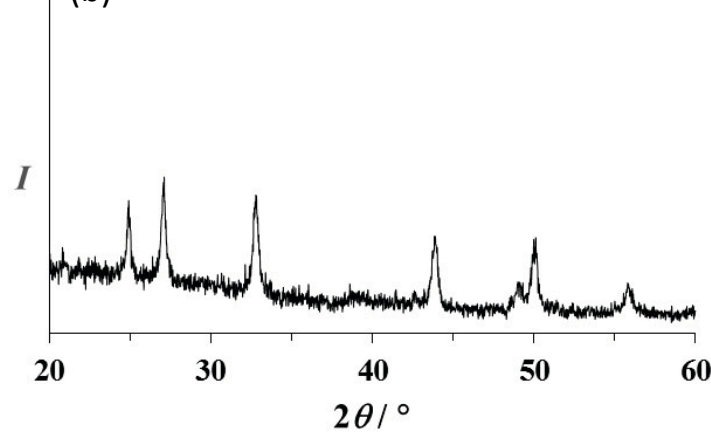

Figure 1. (a) Scanning electron micrographs of the vaterite crystal seeds used for inoculation of supersaturated calcium carbonate solutions. (b) X-ray powder diffraction pattern of the vaterite crystal seeds, which shows only diffraction peaks of vaterite. 


\section{Crystal Growth Kinetics}

Figure 2. shows the progress curves, $\mathrm{pH}$ versus time, obtained from crystal growth experiments in control system: $c_{\mathrm{i}}\left(\mathrm{CaCl}_{2}\right)=c_{\mathrm{i}}\left(\mathrm{Na}_{2} \mathrm{CO}_{3}\right)=1.0 \times 10^{-3} \mathrm{~mol} \mathrm{dm}^{-3}$, initial supersaturation with respect to vaterite, $S_{\mathrm{v}, 0}=3.7$ and the mass concentration of vaterite seeds, $\rho_{\text {vaterite }}=250 \mathrm{mg} \mathrm{dm}^{-3}$. The measured $\mathrm{pH}$ started to continuously decrease as a consequence of the $\mathrm{CaCO}_{3}$ precipitation $\left(\mathrm{Ca}^{2+}\right.$ and $\mathrm{CO}_{3}{ }^{2-}$ removal from solution caused the changes of ionic equilibria) immediately after the inoculation of the supersaturated systems. The good superimposition coincidence (overlapping) among all experimental curves indicates satisfying reproducibility, necessary for evaluation of the kinetic parameters and determination of growth mechanisms.

Figure 3. shows the total concentrations of dissolved calcium species, $c_{\text {tot }}$, calculated from the measured $\mathrm{pH}$, as a function of time, $t$. The progress curves obtained in control systems are shown together with the respective systems containing different concentrations of pAsp, pGlu or pLys. It is evident that the increase of the acidic poly(amino acid) concentrations ( $\mathrm{pAsp}$ and pGlu), caused a progressive lowering of the slope of respective curves with respect to model systems, which indicates the growth rate inhibition. In addition, the increasing additive concentrations caused the termination of the vaterite growth at different concentrations of dissolved $\mathrm{CaCO}_{3}$ : the effect of pAsp being more pronounced than for pGlu. However, no inhibition and termination of the crystal growth was observed in the presence of plys, even at concentrations as high as, $c=4$ ppm: all progress curves coincide with that of the control system.

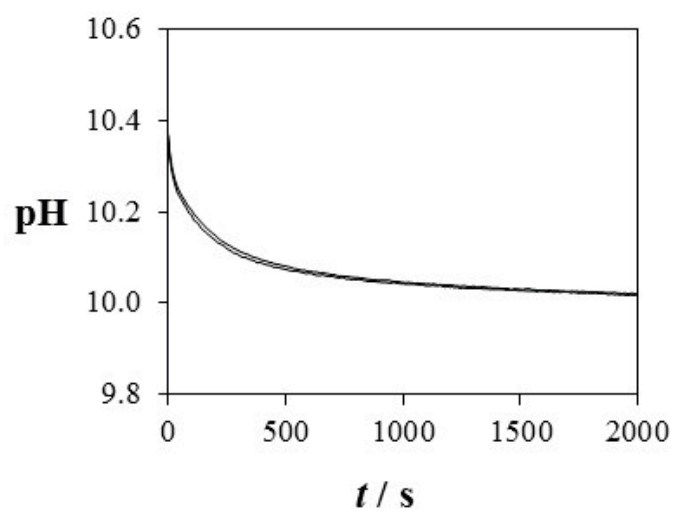

Figure 2. Progress curves, $\mathrm{pH}$ versus time, of the seeded vaterite growth in the control precipitation system $\left(T=298 \mathrm{~K}\right.$ and $c_{\mathrm{i}}\left(\mathrm{CaCl}_{2}\right)=c_{\mathrm{i}}\left(\mathrm{Na}_{2} \mathrm{CO}_{3}\right)=1.0 \times 10^{-3} \mathrm{~mol} \mathrm{dm}^{-3}$, initial supersaturation, $S_{v, 0}=3.7$, vaterite seed mass concentration, $\rho_{\text {vaterite }}=250 \mathrm{mg} \mathrm{dm}^{-3}$ ). The overlapping of the curves indicates a good reproducibility of the experiments.
In order to evaluate the mechanism which predominantly controls the process of vaterite crystal growth for a given range of supersaturations, the analysis of the growth rates have been made. Despite the fact that the so-called "nonclassical" description of crystal nucleation and growth of $\mathrm{CaCO}_{3}$ is extensively used in literature, the progress curves found in this work indicate that the growth of vaterite
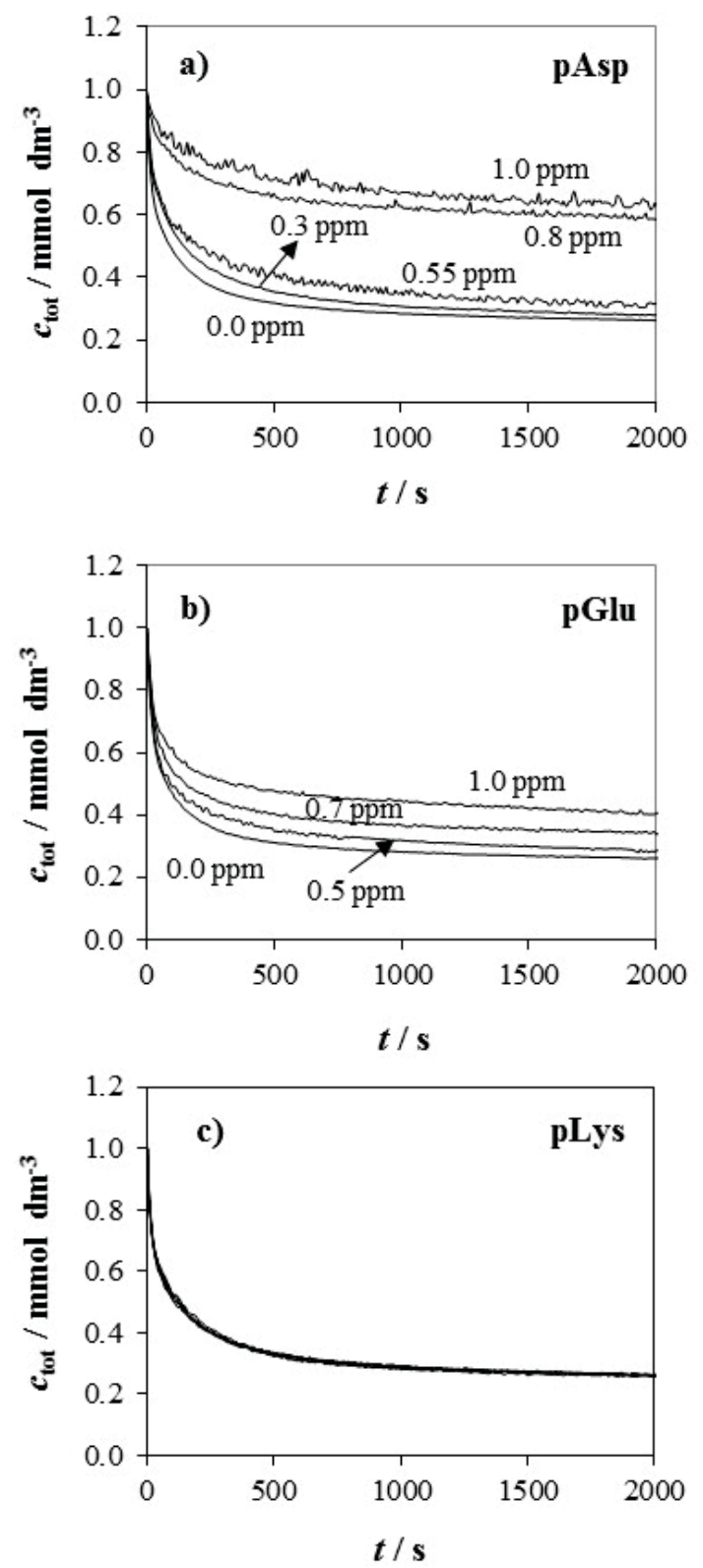

Figure 3. Progress curves, ctot versus time, of the seeded vaterite growth in the control systems and in the systems containing different concentrations of: (a) pAsp; (b) pGlu and (c) pLys $\left(0.2 \mathrm{ppm}<c_{\mathrm{i}}\right.$ (pLys) $\left.<4.0 \mathrm{ppm}\right)$. 

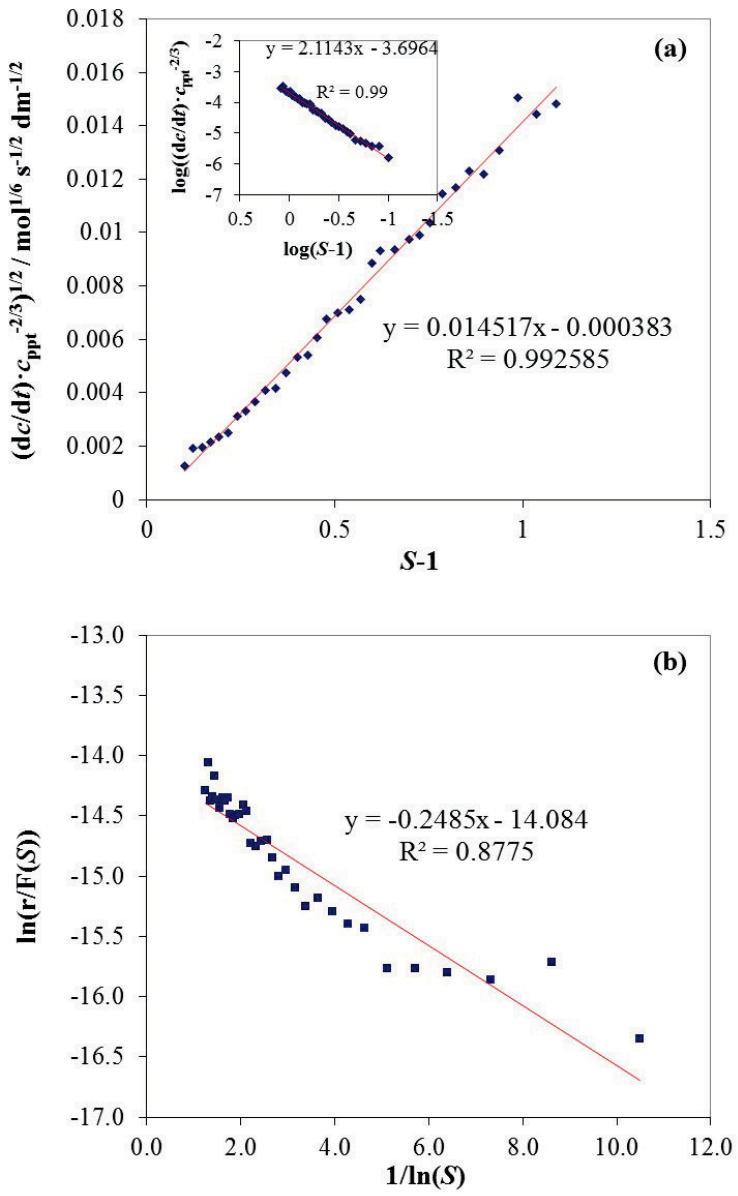

Figure 4. Test plots for the parabolic (a) and exponential (b) rate laws for the seeded vaterite crystal growth in the control precipitation system. Inset in (a) shows logarithmic plot of the growth rate vs relative supersaturation for the precipitation system giving slope $n=2.11$ (empirical rate order). particles can be fully described with the classical growth theory. ${ }^{[50]}$ Namely, in this particular case, layer-by-layer deposition of growth units $\left(\mathrm{Ca}^{2+}\right.$ and $\left.\mathrm{CO}_{3}{ }^{2-}\right)$ on the top of the already formed crystal planes of vaterite seed, can be correlated to the measured continuous changes of their concentrations in the solution (calculated from $\mathrm{pH}$ changes and confirmed with chemical analyses). Thus, the growth rates, $v_{\mathrm{g}} \equiv \mathrm{d} c / \mathrm{d} t$, corrected for the changes of the surface area of vaterite seeds, $c_{\mathrm{ppt}}{ }^{2 / 3}$, were plotted as a function of the supersaturation, $S-1$ in order to test different theoretical models. Figure 4 . shows the test plots for the parabolic, $\mathrm{d} c / \mathrm{d} t=k_{2} c_{\mathrm{ppt}}{ }^{2 / 3}(S-1)$, and exponential, $\mathrm{d} c / \mathrm{d} t=$ $k_{\mathrm{e}} S^{7 / 6}(S-1)^{2 / 3}(\ln S)^{1 / 6} \exp \left[-K_{\mathrm{e}} / \ln S\right]=k_{\mathrm{e}} F(S) \exp \left[-K_{\mathrm{e}} / \ln S\right]$, rate laws in the control system. Indeed, the straight line obtained for the parabolic rate law indicates that under given conditions vaterite growth was controlled by a second order surface reaction, i.e. by the addition of growth units into a spiral step at the crystal screw dislocations (Figure 4a) ${ }^{[44-46]}$. The inset in Figure 4a shows that the respective logarithmic plot, $\log (\mathrm{d} c / \mathrm{d} t)$ versus log $(S-1)$, gave the slope, $n=2.11$, which represents the empirical rate order of the reactions. On the other hand, the vaterite crystal growth rate constants were determined from the slopes of the straight lines for the parabolic test plots, $k_{2}=2.13 \times 10^{-4} \pm 0.22 \times 10^{-4} \mathrm{~mol}^{1 / 3} \mathrm{dm}^{-1} \mathrm{~s}^{-1}$. The spiral growth mechanism obtained in this work, as well as the respective constants, are consistent with some previous findings. ${ }^{[46]}$

Similarly to the control, the appropriate tests were performed in the systems with the addition of selected poly(amino acids) (pAsp, pGlu). However, the progressive growth rate inhibition was demonstrated even before as a lowering of the slope of the progress curves (Figure 3 ), while the respective test plots showed that at the highest concentrations of pAsp (0.8 and $1 \mathrm{ppm})$ and pGlu (0.7 and

Table 1. Vaterite crystal growth kinetic parameters for control system and systems with the addition of poly(amino acids): initial poly(amino acid) concentrations $\left(c / p p m\right.$ and $c / \mu \mathrm{mol} \mathrm{dm}^{-3}$ ), reaction order $(n)$, parabolic growth rate constant $\left(k_{2}\right)$ and exponential growth rate constant $\left(K_{\mathrm{e}}\right)$.

\begin{tabular}{|c|c|c|c|c|c|}
\hline System & $c / p p m$ & $c / \mu \mathrm{mol} \mathrm{dm} \mathrm{m}^{-3}$ & $n$ & $10^{4} \mathrm{k}_{2} / \mathrm{mol}^{1 / 3} \mathrm{dm}^{-1} \mathrm{~s}^{-1}$ & $K_{\mathrm{e}}$ \\
\hline Control & 1 & / & 2.11 & 2.13 & \\
\hline \multirow{3}{*}{ pGlu } & 0.5 & 3.87 & 2.30 & 2.10 & \\
\hline & 0.7 & 5.42 & 4.00 & & -1.84 \\
\hline & 1.0 & 7.75 & 5.45 & & -3.89 \\
\hline \multirow{4}{*}{ pAsp } & 0.3 & 2.61 & 2.26 & 1.77 & \\
\hline & 0.55 & 4.78 & 2.42 & 1.66 & \\
\hline & 0.8 & 6.95 & 7.85 & & -10.04 \\
\hline & 1.0 & 8.69 & 8.37 & & -11.14 \\
\hline \multirow{4}{*}{ pLys } & 0.2 & 1.56 & 2.33 & 1.90 & \\
\hline & 0.5 & 3.90 & 2.12 & 2.02 & \\
\hline & 1.0 & 7.80 & 2.27 & 1.92 & \\
\hline & 4.0 & 31.21 & 2.22 & 1.96 & \\
\hline
\end{tabular}


$1 \mathrm{ppm}$ ) the changes of the controlling mechanism occurred as well. Thus, Figure 5 shows that the screw dislocation controlled growth obtained in the model systems and at lower additive concentrations, changed to surface nucleation controlled growth when concentration of additives exceeded a certain critical values. The observed changes of the vaterite growth mechanism, from the growth on spiral step, to the surface nucleation, may be somewhat unexpected, but they could be explained if the existence of two parallel growth processes (spiral growth and surface nucleation) are assumed. Namely, in a pure systems, when the overall growth rate is a sum of such individual contributions, the faster (spiral growth) becomes the rate determining process. However, the selective adsorption of poly(amino acid) molecules on the active kinks at the spiral steps can create conditions at which the surface nucleation is the only growth mechanism. The results of kinetic measurements in the control systems and in the systems with the addition of the poly(amino acids) are summarized in Table 1. It should be noted that the addition of pLys, in the whole range of concentrations applied, did not cause significant growth inhibition (rate constant, $k_{2}$ ) or change of the vaterite growth mechanism (reaction order, $n$ ).

The formation mechanism of vaterite in the presence of poly(amino acids) and the mode of poly(amino acid) interaction with the vaterite surface have been estimated in our previous publication in which spontaneous precipitation has been studied. ${ }^{[35]}$ For that purpose, the morphological and structural analyses, supported with the electrokinetic mobility measurements of vaterite seeds have been made. The results showed that the $\zeta$-potential of vaterite crystals suspended in the saturated $\mathrm{CaCO}_{3}$ solution was negative $(-4 \mathrm{mV} \leq \zeta \leq-26 \mathrm{mV})$ within the whole $\mathrm{pH}$ range $(9.0 \leq \mathrm{pH} \leq 10.6)$, while the addition of 2 ppm pAsp or 2 ppm pGlu caused the $\zeta$ potential to become even more negative. However, the addition of plys changed the $\zeta$-potential to positive, which indicates that the selected poly(amino acids) are adsorbed on the surface of vaterite. At that, it was assumed that the acidic poly(amino acids) interacted with their carboxylate groups specifically with the calcium ions at the vaterite surface, while the plys interactions are electrostatic and nonspecific $(\mathrm{p} / \mathrm{pAsp}=1.8, \mathrm{p} / \mathrm{pGlu}=2.1, \mathrm{p} / \mathrm{pLys}=12.3)$. Therefore, for the additional and deeper insight into the mode of interactions between the vaterite crystals and selected poly(amino acids), the analyses of the crystal growth kinetics of previously prepared and well characterized vaterite particles, have been applied in this work. Figure 6 shows the dependence of vaterite growth rate, $\mathrm{d} c / \mathrm{d} t$, corrected for the change in the surface area of vaterite seeds $\left(c_{\mathrm{ppt}}{ }^{-2 / 3}\right)$, on relative supersaturation, $S-1$, for $\mathrm{pAsp}$, pGlu and pLys added into the precipitation systems.
Evidently, acidic poly(amino acids) slowed down the crystal growth rate of vaterite. In addition, it could be seen that the highest concentrations of pAsp and pGlu caused the termination of vaterite growth (dead zone) at certain critical supersaturation, $S^{*}: S^{*}=2.4$ at $c(\mathrm{pAsp})=1.0 \mathrm{ppm}$; $S^{*}=2.25$ at $c(\mathrm{pAsp})=0.8 \mathrm{ppm} ; S^{*}=2.3$ at $c(\mathrm{pGlu})=1 \mathrm{ppm} ;$ $S^{*}=1.5$ at $c(\mathrm{pGlu})=0.7 \mathrm{ppm}$. According to the generally accepted models that describe the impact of impurities on the crystal growth kinetics ${ }^{[51,52]}$ the critical supersaturation is a value below which a crystal does not grow. That is, when
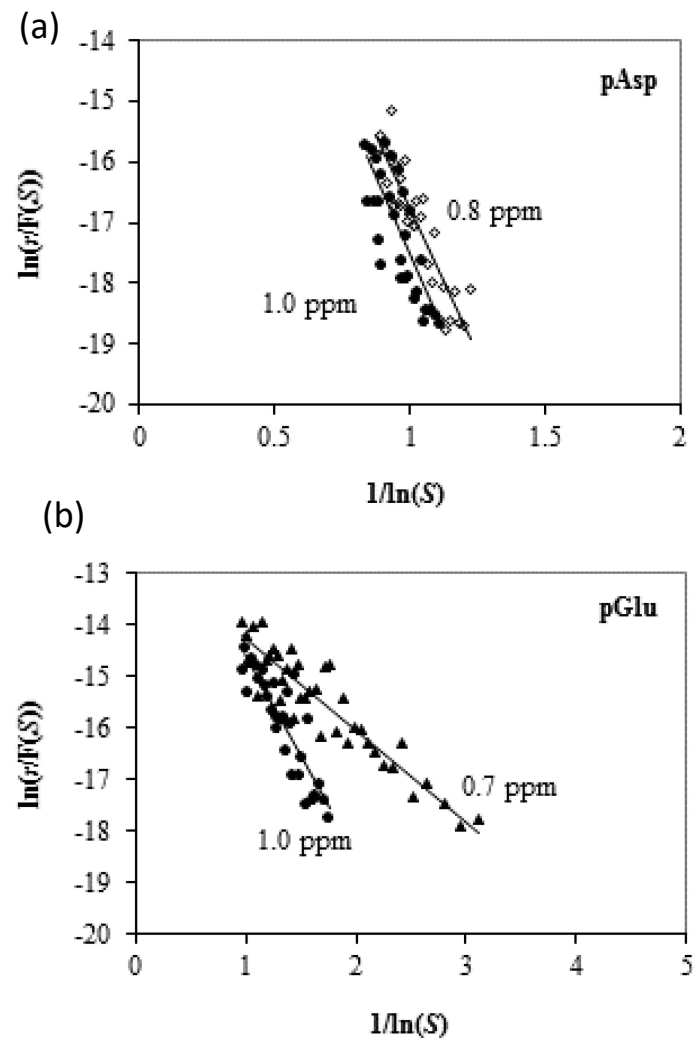

(c)

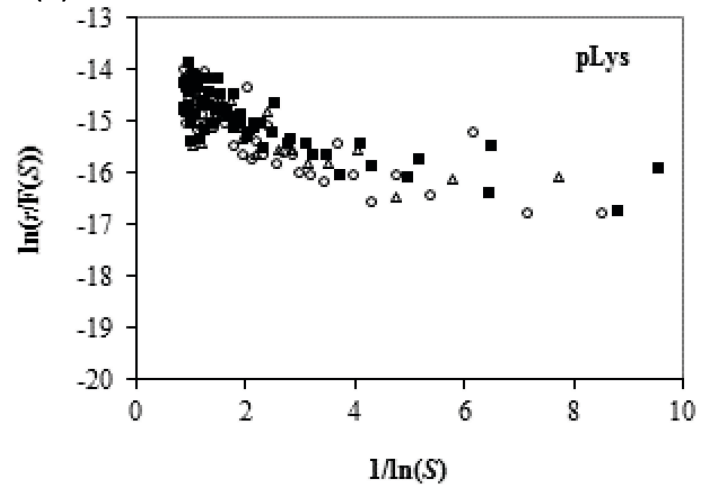

Figure 5. Test plots for the exponential rate law for the seeded vaterite crystal growth in the systems in which specific concentrations of (a) pAsp; (b) pGlu and (c) pLys $\left(0.2 \mathrm{ppm}<c_{i}(\right.$ pLys $\left.)<4.0 \mathrm{ppm}\right)$ were present. 
the advancing step of the growing crystal contacts the adsorbed additive, it will be stopped at the supersaturation at which the distance between the adsorbed molecules is smaller than the diameter of the respective surface nucleus. On contrary, when the distance is larger than the diameter, the step will curl around the additive molecules, slow down the growth, and change crystal morphology.

Additional analysis of the mechanisms of poly(amino acid) effects on the growth of vaterite crystals was performed by applying Kubota and Mullin's model, [53] which enable a quantitative estimation of the effects of additives on the crystal growth rate:

$$
v / v_{0}=1-[\alpha K c /(1+K c)]
$$

In this expression, $v / v_{0}$ is the relative growth rate of a step on the crystal surface and can be replaced with the relative crystal growth rate, which are supposed to be correlated. The value $v$ corresponds to the system containing the additive and $v_{0}$ to the control system. The expression (4) implicates that the crystal growth rate in the presence of the additive is a function of the crystal surface coverage, $\vartheta_{\text {eq }}$ $=K c /(1+K c)$, and the additive effectiveness, $\alpha$ ( $K$ is the Langmuir constant and $c$ is the additive concentration in the solution, expressed in $\mu \mathrm{mol} \mathrm{dm}^{-3}$ ). Indeed, the effectiveness can be perceived as the stereochemical difference between different impurity molecules. Figure 7. shows the plots of the relative crystal growth rate reduction for vaterite seeds, $v / v_{0}$, at different relative supersaturations, $(S-1)$, as a function of different poly(amino acid) concentrations. In the case of pAsp or pGlu, the relative growth rates, $v / v_{0}$, significantly decrease (roughly linearly) to zero, with increasing the respective poly(amino acid) concentrations. On contrary the changes caused by pLys are not significant, indicating just minor inhibition (reduction) of growth rate for the given range of concentrations. The observed different activities of pLys in comparison to pAsp and pGlu,

Table 2. Adsorption parameters deduced from the vaterite crystal growth kinetics data and according to Kubota and Mullin's model: $K_{\text {ad }}$ is the Langmuir adsorption constant and $\alpha$ is the effectiveness factor.

\begin{tabular}{cccc}
\hline & $S-1$ & $K_{\mathrm{ad}} / \mathrm{dm}^{3} \mathrm{~mol}^{-1}$ & $\alpha$ \\
\hline \multirow{3}{*}{ pAsp } & 1.2 & & 8.8 \\
& 1.6 & & 8.5 \\
& 2.0 & 15730 & 7.6 \\
& 2.4 & & 5.0 \\
pGlu & 1.2 & & \\
& 1.6 & 31750 & 4.6 \\
& 2.0 & & 3.9 \\
\hline
\end{tabular}

could be related to its weak, nonselective bonding (probably electrostatic) at the exposed crystal surfaces. ${ }^{[34,35]}$ It should be noted that conclusions are complementary to those obtained from analyses of the kinetics data (Figure 6). The solid lines drawn through the experimental points in Figure 7 are obtained by fitting the respective set of data with the function given by [Eq. (4)].

However, the respective values of the adsorption constants $K_{\text {ad }}$ calculated by applying the Kubota and Mullin's model (Table 2) are significantly higher in the case of pGlu, thus indicating a higher affinity of pGlu for vaterite surface. On contrary, the effectiveness factors, $\alpha$, in case of
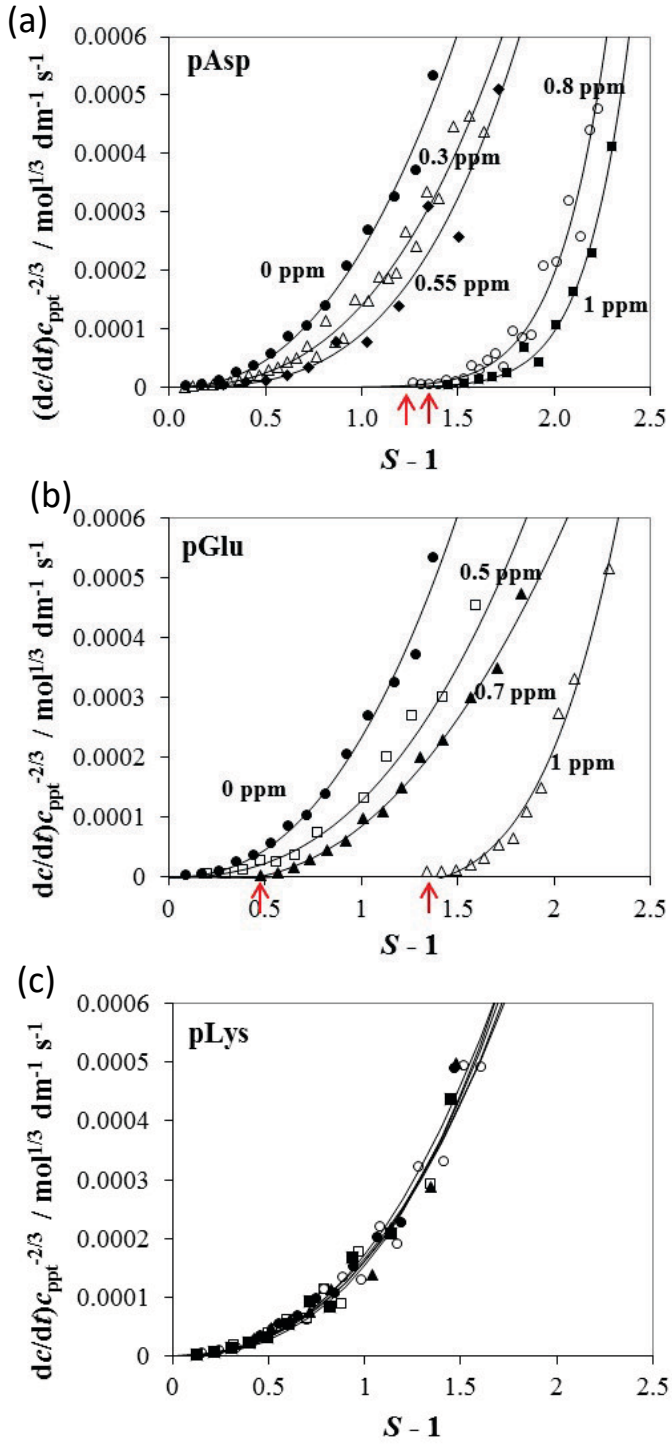

Figure 6. Crystal growth rate of vaterite seed as a function of relative supersaturation in control system and in the systems with different concentrations of pAsp, pGlu and pLys $\left(0.2 \mathrm{ppm}<c_{\mathrm{i}}(\mathrm{pLys})<4.0 \mathrm{ppm}\right)$. Critical supersaturations are indicated. 
both, pAsp and pGlu, decrease with increasing the supersaturation and is higher than 1 . According to the model, when $\alpha>1$ the reduction of the crystal growth rate, and even a complete inhibition at higher additive concentrations, points to very effective additive, which strongly and specifically adsorb on the active growth sites.

Previously, ${ }^{[34]}$ the same range of additive concentrations has been applied in the calcite seed systems, but the observed differences between pAsp and

a)
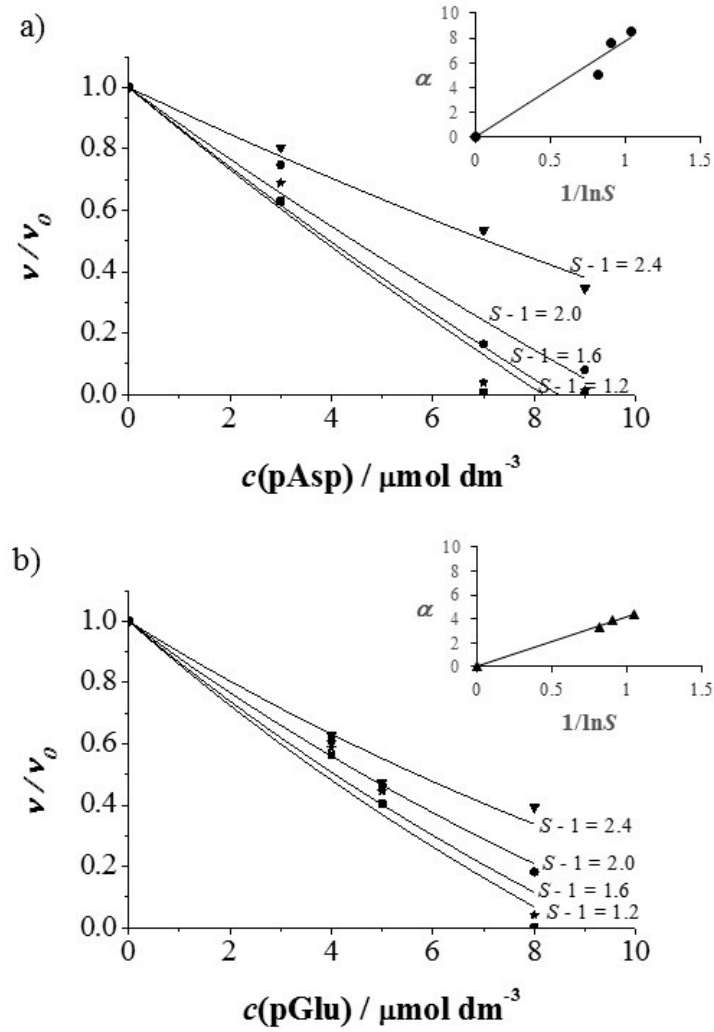

c)

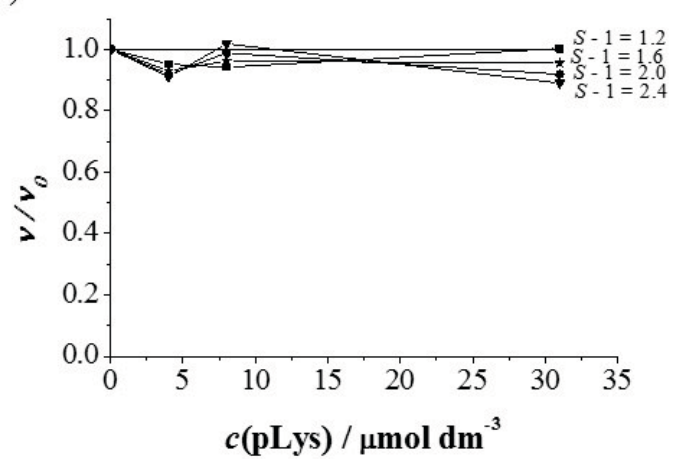

Figure 7. Relative growth rate of vaterite seed crystals at different supersaturations, expressed as a function of (a) pAsp; (b) pGlu and (c) pLys concentrations. Insets show effectiveness factor of the corresponding additives as a function of the reciprocal of supersaturation.
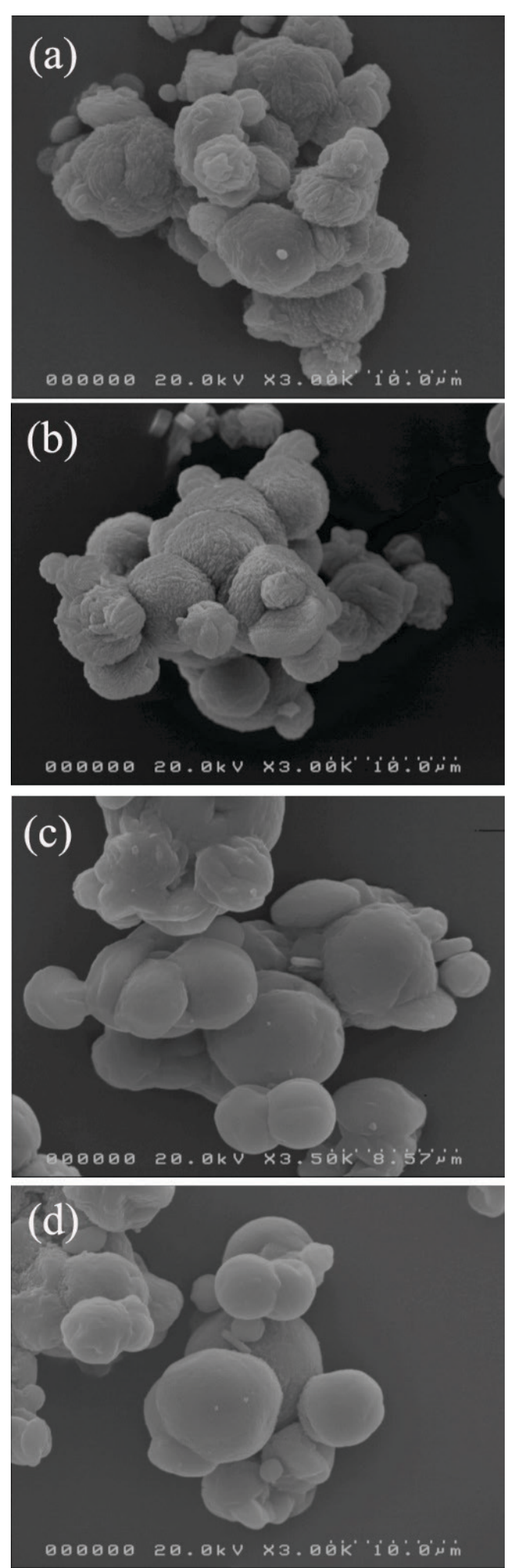

Figure 8. Scanning electron micrographs of vaterite seed crystals (a), and vaterite crystals after overgrowth experiments in the control system (b) and in the presence of additives: (c) 0.3 ppm pAsp and (d) 0.7 ppm pGlu. 
pGlu impact on growth were more pronounced and the effect of pAsp was stronger. Due to the noteworthy chemical similarity between pGlu and pAsp, explanation for their different interactions with different mineral surfaces and morphologies, can be attributed to secondary structures that these molecules assume in a solution containing $\mathrm{Ca}^{2+}$. Thus pAsp tends to adopt more extended regions in $\beta$ pleated sheet conformation, whereas pGlu adopts predominantly $\alpha$-helix and random coil conformations and just to a lesser extent the $\beta$-pleated sheet conformation. ${ }^{[23]}$ In the case of calcite this extended $\beta$-sheet regions enable more coordination bonds between side chain carboxylate groups and calcium ions at the calcite surface. On contrary, in the case of vaterite and the observed preferential adsorption of pGlu, the higher conformational flexibility of pGlu favours the interaction with different stable crystalline planes of vaterite. ${ }^{[54]}$

\section{Morphology}

The morphological changes of vaterite seed that occurred during the crystal growth in the control precipitation system as well as in the systems containing poly(amino acids) were observed by the SEM. Figure 8 shows SEM micrographs of typical vaterite seed crystals (Figure 7.a) as well as the overgrown vaterite crystals in the control system (Figure $7 \mathrm{~b}$ ) and in systems with the added pAsp, $c=0.3 \mathrm{ppm}$ (Figure 7c) and pGlu, $c=0.7 \mathrm{ppm}$ (Figure 7d). Vaterite particles in the control system appeared in the form of relatively regular spheres with rough surfaces, but the presence of pAsp and pGlu in the precipitation systems affected their roughness and turned them to relatively smooth spheres. Probably, smoothing of the surfaces is a consequence of the adsorption of pAsp and pGlu at the polycrystalline surfaces and subsequent formation of smaller primary particles.

Since the addition of plys did not cause the dead zone formation or change of morphology of growing vaterite crystals, the indication was that the mode of its interaction with the vaterite surface was different, most likely consistent with those obtained from electrophoretic mobility measurements. ${ }^{[35]}$

\section{CONCLUSIONS}

The crystal growth kinetics of the vaterite has been investigated in the precipitation system in which the initial reactant concentrations were, $c_{i}\left(\mathrm{CaCl}_{2}\right)=c_{i}\left(\mathrm{Na}_{2} \mathrm{CO}_{3}\right)=$ $1.0 \times 10^{-3} \mathrm{~mol} \mathrm{dm}^{-3}$ and $S_{\mathrm{v}, 0}=3.7$. The influence of the acidic polypeptides, pAsp and pGlu, as well as pLys, on the kinetics of vaterite crystal growth has been studied in the same system, in order to reveal the mode of interfacial interactions between mineral surfaces and synthetic macromolecules. The selected macromolecules mimic the natural proteins responsible for biomineralization of mollusc shells. The XRD and FT-IR analysis confirmed that in all experiments only vaterite appeared, thus indicating no spontaneous coprecipitation or epitaxial growth of other solid $\mathrm{CaCO}_{3}$ modifications.

The kinetic data showed that the crystal growth of vaterite was controlled by a second order surface reaction, i.e. the integration of ions into the spiral step emerging from the surface dislocation. The addition of small amounts of pGlu and pAsp inhibited the crystal growth of vaterite, while at the highest applied concentrations, the surface nucleation became the rate controlling mechanism. The addition of pLys did not significantly influence the crystal growth of the vaterite.

The analyses of the kinetic data by applying the Kubota-Mullin's model which correlates the mode of interactions of additives with growing mineral surfaces, indicated stronger adsorption of pGlu onto vaterite, which has been explained with higher conformational flexibility of pGlu molecules and better matching with specific vaterite morphology. Indeed, the adsorption of pAsp and pGlu at the vaterite surfaces was additionally corroborated by the observed changes of morphology of seed particles.

Acknowledgment. This work has been financially supported by the Croatian Science Foundation under the project IP-2013-11-5055.

\section{REFERENCES}

[1] D. L. G. Rowlands, R. K. Webster, Nat. Phys. Sci. 1971, 229, 158.

[2] J. D. C. McConnell, Mineral. Mag. 1960, 32, 535.

[3] G. Falini, S. Fermani, S. Vanzo, M. Miletic, G. Zaffino, Eur. J. Inorg. Chem. 2005, 162.

[4] A. P. Ariani, K. J. Wittmann, E. Franco, Biol. Bull. 1993, 185, 393.

[5] G. Lambert, C. C. Lambert, Connective Tissue Research 1996, 35, 25.

[6] L. Qiao, Q. L. Feng, Y. Liu, Mater. Lett. 2008, 62, 1793.

[7] L. Kabalah-Amitai, B. Mayzel, Y. Kauffmann, A. N. Fitch, L. Bloch, P. U. P. A. Gilbert, B. Pokroy, Science 2013, 340, 454.

[8] J.-P. Andreassen, J. Cryst. Growth 2005, 274, 256.

[9] G. Falini, Int. J. Inorg. Mater. 2000, 2, 455.

[10] H. Cölfen, Curr. Opin. Colloid Interface Sci. 2003, 8, 23.

[11] S. Mann, J. Inorg. Biochem. 1986, 28, 363.

[12] S. Mann, D. D. Archibald, J. M. Didymus, T. Douglas, B. R. Heywood, F. C. Meldrum, N. J. Reeves, Science 1993, 261, 1286.

[13] J. Aizenberg, J. Hanson, T. F. Koetzle, S. Weiner, L. Addadi, J. Am. Chem. Soc. 1997, 119, 881.

[14] H. H. Teng, P. M. Dove, C. A. Orme, J. J. De Yoreo, Science 1998, 282, 724. 
[15] Y. Levi, S. Albeck, A. Brack, S. Weiner, L. Addadi, Chem. A Eur. J. 1998, 4, 389.

[16] G. Falini, S. Fermani, M. Gazzano, A. Ripamonti, Chem. A Eur. J. 1998, 4, 1048.

[17] C.-S. Choi, Y.-W. Kim, Biomaterials 2000, 21, 213.

[18] J. Wang, Y. Xu, Y. Zhao, Y. Huang, D. Wang, L. Jiang, J. Wu, D. Xu, J. Cryst. Growth 2003, 252, 367.

[19] W. T. Hou, Q. L. Feng, J. Cryst. Growth 2003, 258, 402.

[20] I. W. Kim, J. L. Giocondi, C. Orme, S. Collino, J. S. Evans, Cryst. Growth Des. 2008, 8, 1154.

[21] K. Delak, J. Giocondi, C. Orme, J. S. Evans, Cryst. Growth Des. 2008, 8, 4481.

[22] A. Hernández-Hernández, A. B. Rodríguez-Navarro, J. Gómez-Morales, C. Jiménez-Lopez, Y. Nys, J. M. García-Ruiz, Cryst. Growth Des. 2008, 8, 1495.

[23] L. Addadi, J. Moradian, E. Shay, N. G. Maroudas, S. Weiner, Proc. Natl. Acad. Sci. U. S. A. 1987, 84, 2732.

[24] A. M. Belcher, X. H. Wu, R. J. Christensen, P. K. Hansma, G. D. Stucky, D. E. Morse, Nature 1996, 381, 56.

[25] S. Albeck, J. Aizenberg, L. Addadi, S. Weiner, J. Am. Chem. Soc. 1993, 115, 11691.

[26] L. Addadi, D. Joester, F. Nudelman, S. Weiner, Chem. A Eur. J. 2006, 12, 980.

[27] J. Aizenberg, J. Hanson, T. F. Koetzle, L. Leiserowitz, S. Weiner, L. Addadi, Chem. A Eur. J. 1995, 1, 414.

[28] J. Aizenberg, L. Addadi, S. Weiner, G. Lambert, Adv. Mater. 1996, 8, 222.

[29] S. Raz, P. C. Hamilton, F. H. Wilt, S. Weiner, L. Addadi, Adv. Funct. Mater. 2003, 13, 480.

[30] Y. Yamamoto, T. Nishimura, A. Sugawara, H. Inoue, H. Nagasawa, T. Kato, Cryst. Growth Des. 2008, 8, 4062.

[31] B.-A. Gotliv, N. Kessler, J. L. Sumerel, D. E. Morse, N. Tuross, L. Addadi, S. Weiner, ChemBioChem 2005, 6, 304.

[32] B.-A. Gotliv, L. Addadi, S. Weiner, ChemBioChem 2003, 4, 522.

[33] L. A. Gower, D. A. Tirrell, J. Cryst. Growth 1998, 191, 153.

[34] B. Njegić-Džakula, L. Brečević, G. Falini, D. Kralj, Cryst. Growth Des. 2009, 9, 2425.
[35] B. Njegić-Džakula, G. Falini, L. Brečević, Ž. Skoko, D. Kralj, J. Colloid Interface Sci. 2010, 343, 553.

[36] X. Zhang, Z. Fan, Q. Lu, Y. Huang, D. L. Kaplan, H. Zhu, Acta Biomater. 2013, 9, 6974.

[37] D. Gebauer, A. Volkel, H. Colfen, Science 2008, 322, 1819.

[38] E. M. Pouget, P. H. H. Bomans, J. A. C. M. Goos, P. M. Frederik, G. de With, N. A. J. M. Sommerdijk, Science 2009, 323, 1455.

[39] A. Neira-Carrillo, D. F. Acevedo, M. C. Miras, C. A. Barbero, D. Gebauer, H. Cölfen, J. L. Arias, Langmuir 2008, 24, 12496

[40] D. Kralj, J. Kontrec, L. Brečević, G. Falini, V. NöthigLaslo, Chem. A Eur. J. 2004, 10, 1647.

[41] D. Kralj, L. Brečević, J. Kontrec, J. Cryst. Growth 1997, $177,248$.

[42] B. P. Pichon, P. H. H. Bomans, P. M. Frederik, N. A. J. M. Sommerdijk, J. Am. Chem. Soc. 2008, 130, 4034.

[43] J. Perić, M. Vučak, R. Krstulović, L. Brečević, D. Kralj, Thermochim. Acta 1996, 277, 175.

[44] A. E. Nielsen, Croat. Chem. Acta 1970, 42, 319.

[45] A. E. Nielsen, J. Cryst. Growth 1984, 67, 289.

[46] D. Kralj, L. Brečević, A. E. Nielsen, J. Cryst. Growth 1990, 104, 793.

[47] D. Kralj, L. Brečević, A. E. Nielsen, J. Cryst. Growth 1994, 143, 269

[48] D. Kralj, L. Brečević, Colloids Surfaces A Physicochem. Eng. Asp. 1995, 96, 287.

[49] A. E. Martell, R. M. Smith, Critical stability constants. Volume 1: Amino acids, Plenum Press, New York and London, 1974.

[50] K. K. Sand, J. D. Rodriguez-Blanco, E. Makovicky, L. G. Benning, S. L. S. Stipp, Cryst. Growth Des. 2012, 12, 842.

[51] K. Sangwal, Prog. Cryst. Growth Charact. Mater. 1996, 32, 3.

[52] N. Cabrera, D. A. Vermilyea, in Growth and Perfection of Crystals, (Eds.: R. H. Doremus, B. W. Roberts, D. Turnbull), Wiley, New York, 1958, pp. 393-410.

[53] N. Kubota. J. W. Mullin, J. Cryst. Growth 1995, 152, 203.

[54] H. J. Meyer, Zeitschrift fur Krist. - Cryst. Mater. 1969, 128, 183. 